Gut and Liver, Vol. 13 No. 4, July 2019, pp. 415-420

\title{
A Newly Designed 3-Dimensional Printer-Based Gastric Hemostasis Simulator with Two Modules for Endoscopic Trainees (with Video)
}

\author{
Dong Seok Lee ${ }^{1,2}$, Ji Yong Ahn², and Gin Hyug Lee ${ }^{2}$ \\ ${ }^{1}$ Department of Gastroenterology, SMG-SNU Boramae Medical Center, Seoul National University of College of Medicine, and ${ }^{2}$ Department of \\ Gastroenterology, Asan Medical Center, University of Ulsan College of Medicine, Seoul, Korea
}

Background/Aims: We used 3-dimensional (3D) printing technology to create a new hemostasis simulator for the stomach and investigated its efficacy and realism in endoscopic hemostasis training. Methods: A new stomach hemostasis simulator, with two hemostasis modules for hemoclipping and injection, was constructed using a 3D printer. Twenty-one endoscopists, including 11 first-year fellows (beginner group) and 10 faculty members (expert group), tested the performance of the simulator. We recorded and reviewed five training sessions and evaluated the simulator with questionnaires using a 7-point Likert scale. Results: The mean evaluation score of the expert group was $6.3 \pm 0.5$ for the hemoclipping module and $6.0 \pm 0.6$ for the injection module. The expert group strongly agreed that endoscopic handling in the simulator was realistic and reasonable for hemostasis training. The mean procedure time for hemoclipping was $72.7 \pm 7.1$ seconds for the beginner group and $19.7 \pm 1.2$ seconds for the expert group. The mean procedure time for injection was $92.1 \pm 9.8$ seconds for the beginner group and $36.3 \pm 2$ seconds for the expert group. The procedure time of beginner group became shorter with repetition and was significantly lower by the fifth trial. Conclusions: A new 3D-printed hemostasis simulator is capable of hemostasis training and can very effectively train beginners before they perform the procedure in patients with gastrointestinal bleeding. (Gut Liver 2019;13:415-420)

Key Words: Printing, three-dimensional; Endoscopy; Simulator; Hemostasis; Education

\section{INTRODUCTION}

The annual incidence of upper gastrointestinal (GI) bleeding ranges from 48 to 160 cases per 100,000 adults, with mortality rates as high as $10 \%$ to $14 \% .{ }^{1}$ Despite advances in endoscopy and medical treatment, the mortality rates of GI bleeding have been largely unchanged in recent decades. ${ }^{2}$ Moreover, GI bleeding can be a life-threatening event in older patients with comorbidities and can thus represent a considerable problem in an aging society. Among several endoscopic techniques which are available for hemostasis, epinephrine injection is the easiest and effective approach but rebleeding occurs in many cases. Therefore, to reduce the risk of rebleeding, it should be combined with another method such as hemoclipping and/or thermocoagulation. Many studies and meta-analyses have examined the efficacy of the available endoscopic techniques in patients with high-risk lesions. ${ }^{3,4}$

Although technical skill and endoscopic experience are the most important determinants of successful hemostasis, endoscopic experience can typically only be obtained by practice with real bleeding cases. Thus, various simulators have been developed to mimic acute bleeding situations to reduce the burden on the practitioner and to improve patient safety. However, they are not actively used because of various disadvantages compared with real situations. The first to be introduced were computer simulators for colonoscopy training, with more recent development of various powerful simulators such as the ENDOX (Medical X Corp., Miami, FL, USA) and GI-Mentor (Simbionix Ltd., Israel). ${ }^{5-7}$ The ENDO-X and GI-Mentor perform various simulations, including endoscopic hemostasis, but these virtual devices are different in elasticity from humans and tactile sensation using hemostatic materials, and expensive to purchase.

Correspondence to: Gin Hyug Lee ${ }^{\mathrm{a}}$ and Ji Yong $\mathrm{Ahn}^{\mathrm{b}}$

Department of Gastroenterology, Asan Medical Center, University of Ulsan College of Medicine, 88 Olympic-ro 43-gil, Songpa-gu, Seoul 05505, Korea

aTel: +82-2-3010-3986, Fax: +82-2-476-0824, E-mail: jhlee409@amc.seoul.kr

'Tel: +82-2-3010-5667, Fax: +82-2-476-0824, E-mail: ji110@hanmail.net

Received on August 31, 2018. Revised on November 1, 2018. Accepted on November 3, 2018. Published online April 17, 2019 pISSN 1976-2283 eISSN 2005-1212 https://doi.org/10.5009/gnl18389

() This is an Open Access article distributed under the terms of the Creative Commons Attribution Non-Commercial License (http://creativecommons.org/licenses/by-nc/4.0) which permits unrestricted non-commercial use, distribution, and reproduction in any medium, provided the original work is properly cited. 
Animal simulators such as pigs and dogs have been used to simulate upper GI hemorrhage ${ }^{8,9}$ but these simulators are limited by their reusability and ethical concerns.

Therefore, to overcome the limitations of the previous endoscopic hemostasis simulators, we developed a new simulator using 3-dimensional (3D) printing technology.

\section{MATERIALS AND METHODS}

\section{Participants}

The study was approved by the Institutional Review Board of Asan Medical Center (IRB number: 2016-0302) and performed in accordance with the principles of the Declaration of Helsinki. Twenty-one endoscopists (10 experts and 11 beginners) were recruited to assess the validity of the simulator in June 2017 at Asan Medical Center, Seoul, Korea. The expert group members were staff with experience of more than 50,000 cases of screening endoscopy and 1,000 cases of hemostasis. Eleven beginner group members were first-year clinical fellows with experience of more than 800 cases of EGD and 200 cases of colonoscopy.

\section{Endoscopic hemostasis simulator development}

The new simulator comprises the main body of the upper GI tract, two hemostasis modules for hemoclipping and injection therapy, and a digital peristaltic pump (EMP-600A; EMS Tech, Yongin, Korea) for supplying artificial blood. The main body of the simulator was modified from previous 3D-printed training simulator for endoscopic biopsy in the stomach (this paper is scheduled to be published). The stereolithographic files of the hard part of the two hemostasis modules and the molds of the soft part were produced with the 3D modeling program, Netfabb professional version 5 (Netfabb GmbH, Lupburg, Germany), and printed with the stereolithography 3D printer, Form 2 (Formlabs Inc, Somerville, MA, USA). The soft part of the hemostasis modules has produced by molding shell technique using the soft silicone, Platsil Gel-10 (Polytek, Easton, PA, USA). After assembly of each part, the two modules were inserted into the two holes of the main body, one on the anterior wall side of the proximal body and the other on the anterior wall side of the antrum. The outer part of each module has two tubes connected to the pump (commercial infusion pump for patients) supplying the artificial blood (Fig. 1). Any conventional endoscopic equipment such as esophagogastroduodenoscopes, hemoclips, and injectors can be used in the new simulator. Our test of endoscopic hemostasis was conducted with a standard single channel endoscope (GIFQ260J; Olympus Co. Ltd., Tokyo, Japan), hemoclip (QuickClip Pro; Olympus Co. Ltd), and injector (NM-400Y-0423; Olympus Co. Ltd). The simulator has designed and produced by an experienced endoscopist.

A hemoclipping module was designed for endoscopic hemoclipping training. The hemoclipping module comprises 16 protruding parts (Fig. 2A). The protruding parts were sealed with
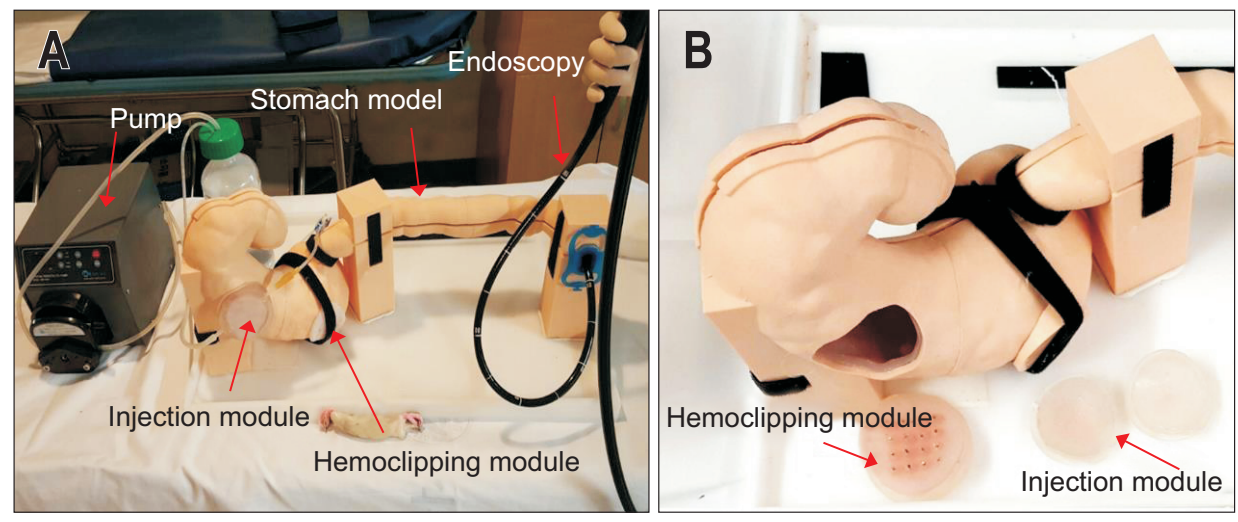

Fig. 1. Hemostasis simulator composed of a hemoclipping module and injection module. (A) Artificial blood is supplied to the simulator through a pump. Any conventional endoscopic equipment can be used. (B) Two hemostasis modules are incorporated into the main body.
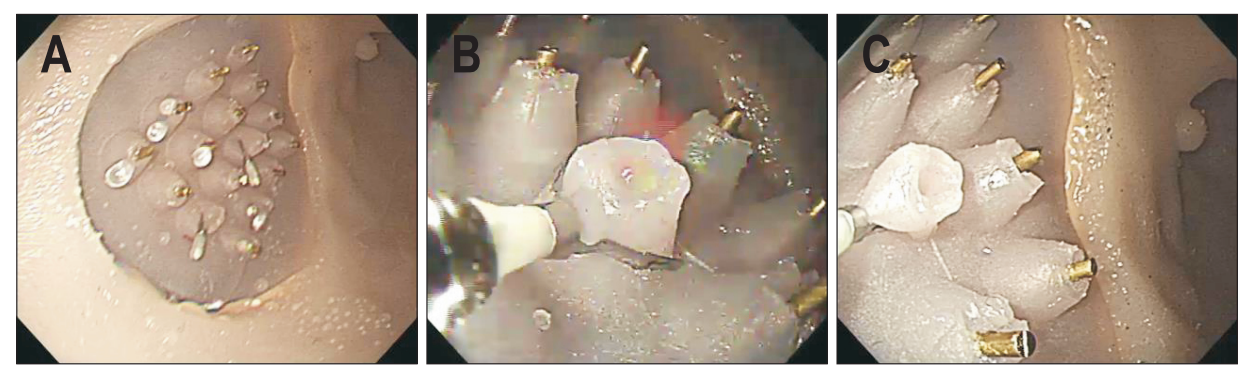

Fig. 2. Hemoclipping module. (A) The hemoclipping module is composed of 16 protruding parts. The protruding parts are sealed with a metal pin blocking the artificial blood. (B) When the metal pin is removed from the protruding hole, artificial blood begins pumping in a manner similar to that of actual blood from a vessel. (C) When the hemoclip is properly positioned, the flow of artificial blood stops. 
a metal pin; removal of the metal pin allows artificial blood to pump in a manner similar to that of real blood from a vessel (Fig. 2B). When the hemoclip is properly positioned, the flow of artificial blood stops (Fig. 2C).

The injection module was designed for the injection of normal saline near the bleeding site. The inner part of the injection module has two stacked chambers and the outer part is made of methacrylic acid ester cured by photo initiator (Fig. 3A). The bleeding pore is connected to the inner chamber and the flow of artificial blood stops when about 2 to $4 \mathrm{~mL}$ of normal saline is injected into the outer chamber (Fig. 3B and C).

\section{Outcome measures}

The primary outcome measure was suitability of the simulator for hemostasis training. The expert group assessed the suitability of the simulator for hemostasis training. Only expert group members were selected as evaluators to rule out as much subjectivity as possible. After testing the simulator, self-administered questionnaires were given to the participants to estimate the efficacy of the hemostasis simulator using a 7-point Likert scale, where 1 indicates poor and 7 indicates excellent. The questions covered the following topics: (1) Handling of simulator is similarity with actual case; (2) Simulator is reasonable for hemostasis training; (3) Working with the simulator will

Table 1. Mean Scores of the Answer to the Question for the Hemostasis Simulator Using a 7-Point Likert Scale

\begin{tabular}{lcc}
\hline \multicolumn{1}{c}{ Index } & Hemoclipping & Injection \\
\hline $\begin{array}{l}\text { Handling of simulator is similarity } \\
\text { with actual case }\end{array}$ & $6.1 \pm 0.4$ & $6.4 \pm 0.5$ \\
$\begin{array}{l}\text { Simulator is reasonable for } \\
\text { hemostasis training }\end{array}$ & $6.0 \pm 0.5$ & $6.4 \pm 0.4$ \\
$\begin{array}{l}\text { Working with the simulator will } \\
\text { improves trainee's skills }\end{array}$ & $6.3 \pm 0.5$ & $6.7 \pm 0.5$ \\
$\begin{array}{l}\text { Training with the simulator reduces } \\
\text { the risk for patients }\end{array}$ & $6.1 \pm 0.7$ & $6.9 \pm 0.5$ \\
$\begin{array}{l}\text { I recommend that the simulator } \\
\text { be used in hemostasis training }\end{array}$ & $6.1 \pm 0.7$ & $6.9 \pm 0.5$ \\
\hline
\end{tabular}

Data are presented as the mean \pm SD. Scale, 1 (strongly disagree) to 7 (strongly agree). improves trainee's skills; (4) Training with the simulator reduces the risk for patients; (5) I recommend that the simulator be used in hemostasis training. The secondary outcome measure was the procedure time for new hemostasis simulator.

\section{Statistical analysis}

Statistical analysis was performed using SPSS software, version 21 (IBM Corp., Armonk, NY, USA). Continuous variables are reported as medians with interquartile ranges and/or as means \pm standard deviations. Descriptive statistics were used to describe data, such as reusability and similarity.

\section{RESULTS}

All participants completed the hemoclipping and injection methods successfully. There were no malfunctions during the test.

\section{Expert group's opinions of the hemostasis simulator}

The 10 experts estimated the realism level of the new hemostasis simulator using a 7-point Likert scale (Table 1). The mean score of the hemoclipping module was $6.3 \pm 0.5$ and the that of the injection module was 6.0 \pm 0.6 . Participants strongly agreed that endoscopic handling in the simulator was realistic and reasonable for endoscopic training. Notably, all of the participants felt that this training could reduce the risk for patients.

\section{Hemostasis simulator outcomes}

Each group tested the hemostasis simulator. To compare the learning effect of the hemostasis simulator, the procedure times of each group were measured five times. Procedure time sharply decreased after each trial in the beginner group (Fig. 4). The mean procedure time per bleeding hole with hemoclipping module was $72.7 \pm 7.1$ seconds for beginner group and 19.7 \pm 1.2 seconds for expert group (Table 2). The number of clips used for the hemostasis was 1 per person. The mean procedure time for the injection module was $92.1 \pm 9.8$ seconds for beginner group and $36.3 \pm 2$ seconds for expert group. The material used for the injection was a mean $4 \pm 1 \mathrm{~mL}$ of normal saline.
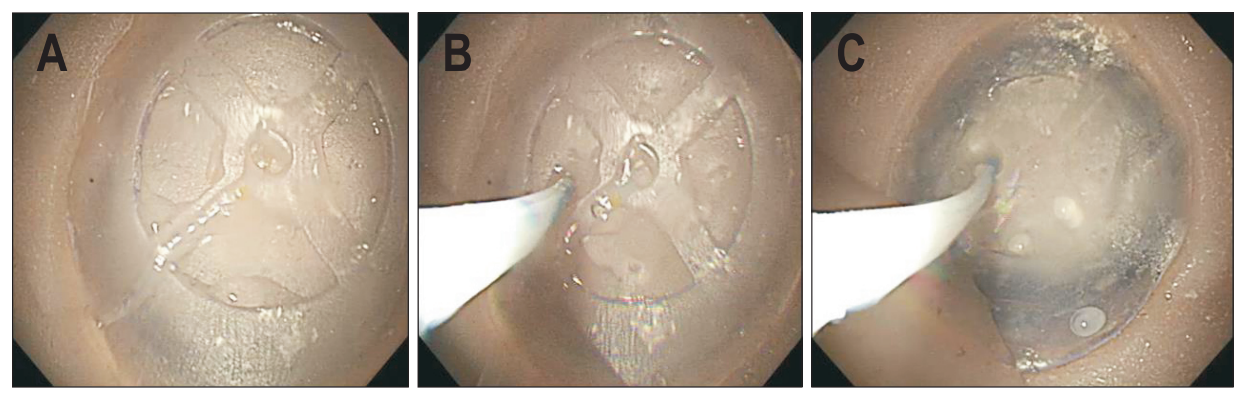

Fig. 3. Injection module. (A) The inner part of the injection module has two stacked chambers and the outer part is made of the methacrylic acid ester cured by photoinitiator. (B, C) When the saline is injected into the outer chamber, the flow of artificial blood is diminished and stops if outer chamber pressure is high enough. 

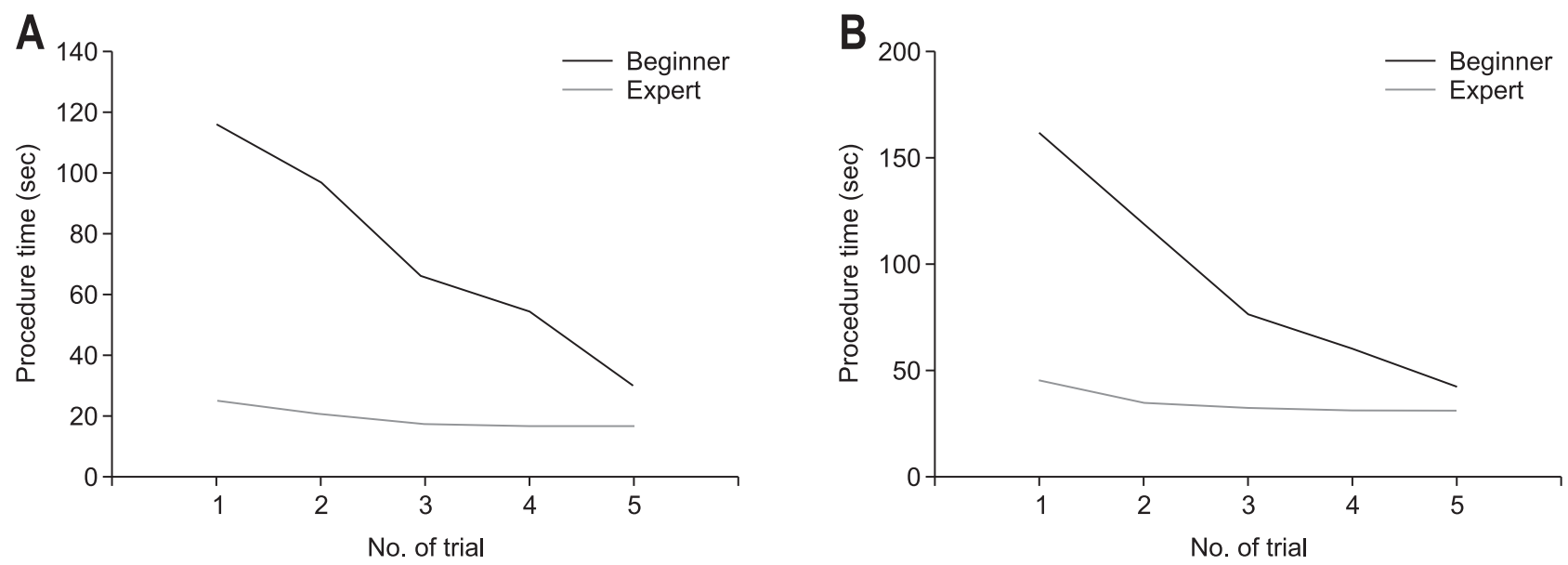

Fig. 4. Procedure times for each trial for each group. (A) Hemoclipping module and (B) injection module.

Table 2. Procedure Completing Times with the New Hemostasis Simulator According to the Experience Level of the Endoscopist

\begin{tabular}{|c|c|c|c|c|}
\hline & \multicolumn{2}{|c|}{ Hemoclipping } & \multicolumn{2}{|c|}{ Injection } \\
\hline & Beginner group $(\mathrm{n}=11)$ & Expert group $(\mathrm{n}=10)$ & Beginner group $(\mathrm{n}=11)$ & Expert group $(\mathrm{n}=10)$ \\
\hline 1st trial & $116.1 \pm 15.3$ & $25.1 \pm 1.2$ & $161.3 \pm 13.4$ & $46 \pm 3.1$ \\
\hline 2nd trial & $96.4 \pm 6.1$ & $21.2 \pm 1.9$ & $118.2 \pm 9.3$ & $36.4 \pm 2$ \\
\hline 3rd trial & $65.8 \pm 3.3$ & $18 \pm 1$ & $77 \pm 10.7$ & $33.6 \pm 1.6$ \\
\hline 4th trial & $54.8 \pm 6.9$ & $17.7 \pm 1$ & $61.1 \pm 8.2$ & $32.9 \pm 2.2$ \\
\hline 5th trial & $30.5 \pm 4.1$ & $16.6 \pm 1$ & $43 \pm 7.2$ & $32.4 \pm 1.5$ \\
\hline Mean of 5 trials & $72.7 \pm 7.1$ & $19.7 \pm 1.2$ & $92.1 \pm 9.8$ & $36.3 \pm 2$ \\
\hline
\end{tabular}

Data are presented as mean \pm SD. Time expressed in seconds.

\section{DISCUSSION}

We developed a new endoscopic hemostasis simulator for hemoclipping and injection. The simulator is fully compatible with conventional endoscopic equipment and can be made from a 3D printer and basic materials. Ten experts evaluated the new simulator and agreed that it is effective for training. The approximate price of the simulator is $\$ 200$, and the price may become lower depending on the production quantity. Because of the low cost and compatibility, endoscopists in hospitals without sufficient experience can easily receive training, which should significantly improve patient safety.

We assessed the efficacy and realism of a novel 3D endoscopic hemostasis simulator, with differing levels of experience. Procedure time became shorter as the participants repeated the training sessions. The expert group strongly agreed that this simulator was a useful tool for improving endoscopic hemostasis skills of trainee without any risks to patients.

Endoscopic hemostasis is a well-established procedure for the treatment of GI bleeding and effectively reduces morbidity and mortality. ${ }^{10}$ In previous studies, animal models have been used in training to simulate upper GI hemorrhage. ${ }^{8,9}$ However, this approach is not widely used due to problems with cost, us- ability, safety, ethics, and reusability. ${ }^{11}$ Because of ethical concerns, realistic ex vivo models to simulate esophageal varices and peptic ulcer hemorrhage have been developed. ${ }^{12,13}$ However, this surgical bio-simulation model also has the same limitations as previous animal models. In 2001, an artificial tissue model, EASIE, was introduced, but it is complex, bulky, and expensive. ${ }^{14}$ Various virtual simulators have recently been introduced but their use is limited due to their cost. ${ }^{15}$ In order to overcome the limitations of previous models such as reliability, cost-effectiveness, and reusability, we thus developed a new simulator and reported the results in this study.

3D printing has been used in various fields of medicine. 3Dprinted materials can be used to train various procedures, such as in orthopedics, spinal surgery, neurosurgery, and cardiac surgery. ${ }^{16,17}$ Recently, a simulator for endoscopic training and biopsy was developed and evaluated in our center. ${ }^{18,19}$ Because this first stomach biopsy simulator was made from a rectangular plastic box, it provides a different feeling compared with the actual stomach. Thus, a second stomach biopsy simulator was made with a 3D printer to have the same shape as the actual stomach. The second stomach biopsy simulator was found to be an effective and realistic method for improving biopsy skills without any risk to patients (unpublished data). Based on this 
new 3D printing technology and our experience, we created a new simulator for bleeding control to overcome the disadvantages of existing simulators.

This study had several limitations of note. First, gastric movement due to the heartbeat and respiration were not reproduced; thus, endoscopic hemostasis would have been easier than in a real patient. Second, there was a possible bias in evaluating the simulator using questionnaires. Third, the level of elasticity is different from real stomach. Nevertheless, our 3D-printed simulator has various benefits for endoscopic hemostasis training and could be the basis for a more complex simulator that overcomes the above drawbacks. Fourth, our simulator was not compared to other simulators and we could not include external expert groups for test. Therefore, there is possibility of subjectivity in the test for this simulator. To overcome this limitation, we tried to consist the evaluators as experts of our center.

In conclusion, the new simulator described herein has advantages over the previous hemostasis simulators due to improved realism, cost-effectiveness, and reusability. We believe that this new simulator could be applied to endoscopic hemostasis training in many centers and we have plans to improve it further by listening to user feedbacks and by including other hemostatic methods such as coagulation and argon plasma coagulation modules.

\section{CONFLICTS OF INTEREST}

No potential conflict of interest relevant to this article was reported.

\section{ACKNOWLEDGEMENTS}

This study was supported by a grant (2016-7021) from the Asan Institute for Life Sciences, Asan Medical Center, Seoul, Korea.

\section{AUTHOR CONTRIBUTIONS}

Study concept and design, data acquisition, data analysis and interpretation, drafting of the manuscript: D.S.L. Critical revision of the manuscript for important intellectual content, obtained funding, administrative, technical, or material support, study supervision: G.H.L., J.Y.A.

\section{ORCID}

Dong Seok Lee

Ji Yong Ahn

Gin Hyug Lee https://orcid.org/0000-0003-2231-0563

https://orcid.org/0000-0002-0030-3744

https://orcid.org/0000-0003-3776-3928

\section{REFERENCES}

1. Quan S, Frolkis A, Milne K, et al. Upper-gastrointestinal bleeding secondary to peptic ulcer disease: incidence and outcomes. World J Gastroenterol 2014;20:17568-17577.

2. Lu Y, Barkun AN, Martel M; REASON investigators. Adherence to guidelines: a national audit of the management of acute upper gastrointestinal bleeding. The REASON registry. Can J Gastroenterol Hepatol 2014;28:495-501.

3. Marmo R, Rotondano G, Piscopo R, Bianco MA, D’Angella R, Cipolletta L. Dual therapy versus monotherapy in the endoscopic treatment of high-risk bleeding ulcers: a meta-analysis of controlled trials. Am J Gastroenterol 2007;102:279-289.

4. Yuan Y, Wang C, Hunt RH. Endoscopic clipping for acute nonvariceal upper-GI bleeding: a meta-analysis and critical appraisal of randomized controlled trials. Gastrointest Endosc 2008;68:339-351.

5. Sedlack RE, Kolars JC. Computer simulator training enhances the competency of gastroenterology fellows at colonoscopy: results of a pilot study. Am J Gastroenterol 2004;99:33-37.

6. Simbionix Inc. Product brochure [Internet]. c2016 [cited 2017 Sep 22]. Available from: https://simbionix.com/simulators/gi-mentor/.

7. Medical-X Inc. Product brochure [Internet]. c2017 [cited 2019 Feb 27]. Available from: https://www.medical-x.com/.

8. Pinkas H, McAllister E, Norman J, Robinson B, Brady PG, Dawson PJ. Prolonged evaluation of epinephrine and normal saline solution injections in an acute ulcer model with a single bleeding artery. Gastrointest Endosc 1995;42:51-55.

9. Michaletz PA, Judge D. Microwave energy compared with heater probe and BICAP in canine models of peptic ulcer hemorrhage. Gastroenterology 1989;97:676-684.

10. Chou YC, Hsu PI, Lai KH, et al. A prospective, randomized trial of endoscopic hemoclip placement and distilled water injection for treatment of high-risk bleeding ulcers. Gastrointest Endosc 2003;57:324-328.

11. Matthes K, Dunkin BJ, Jensen DM. Simulator training in endoscopic hemostasis. Tech Gastrointest Endosc 2011;13:174-182.

12. Hu B, Chung SC, Sun LC, et al. Eagle Claw II: a novel endosuture device that uses a curved needle for major arterial bleeding: a bench study. Gastrointest Endosc 2005;62:266-270.

13. Neumann M, Hochberger J, Felzmann T, Ell C, Hohenberger W. Part 1. The Erlanger endo-trainer. Endoscopy 2001;33:887-890.

14. Hochberger J, Maiss J, Magdeburg B, Cohen J, Hahn EG. Training simulators and education in gastrointestinal endoscopy: current status and perspectives in 2001. Endoscopy 2001;33:541-549.

15. Triantafyllou K, Lazaridis LD, Dimitriadis GD. Virtual reality simulators for gastrointestinal endoscopy training. World J Gastrointest Endosc 2014;6:6-12.

16. AbouHashem Y, Dayal M, Savanah S, Štrkalj G. The application of 3D printing in anatomy education. Med Educ Online 2015;20:29847.

17. Tack P, Victor J, Gemmel P, Annemans L. 3D-printing techniques in a medical setting: a systematic literature review. Biomed Eng 
Online 2016;15:115.

18. Lee S, Ahn JY, Han M, et al. Efficacy of a three-dimensionalprinted training simulator for endoscopic biopsy in the stomach. Gut Liver 2018;12:149-157.
19. Ahn JY, Lee JS, Lee GH, et al. The efficacy of a newly designed, easy-to-manufacture training simulator for endoscopic biopsy of the stomach. Gut Liver 2016;10:764-772. 